MATHEMATICS OF COMPUTATION

Volume 76, Number 259, July 2007, Pages 1219-1242

S 0025-5718(07)01960-6

Article electronically published on January 25, 2007

\title{
CONVERGENCE OF GODUNOV TYPE METHODS FOR A CONSERVATION LAW WITH A SPATIALLY VARYING DISCONTINUOUS FLUX FUNCTION
}

\author{
ADIMURTHI, SIDDHARTHA MISHRA, AND G. D. VEERAPPA GOWDA
}

\begin{abstract}
We deal with single conservation laws with a spatially varying and possibly discontinuous coefficient. This equation includes as a special case single conservation laws with conservative and possibly singular source terms. We extend the framework of optimal entropy solutions for these classes of equations based on a two-step approach. In the first step, an interface connection vector is used to define infinite classes of entropy solutions. We show that each of these classes of solutions is stable in $L^{1}$. This allows for the possibility of choosing one of these classes of solutions based on the physics of the problem. In the second step, we define optimal entropy solutions based on the solution of a certain optimization problem at the discontinuities of the coefficient. This method leads to optimal entropy solutions that are consistent with physically observed solutions in two-phase flows in heterogeneous porous media. Another central aim of this paper is to develop suitable numerical schemes for these equations. We develop and analyze a set of Godunov type finite volume methods that are based on exact solutions of the corresponding Riemann problem. Numerical experiments are shown comparing the performance of these schemes on a set of test problems.
\end{abstract}

\section{INTRODUCTION}

We are interested in the following single conservation law in one space dimension,

$$
\begin{aligned}
u_{t}+(f(k(x), u))_{x} & =0, \\
u(x, 0) & =u_{0}(x),
\end{aligned}
$$

where $u$ is the unknown, $f$ is the flux function and $k$ is a spatially varying, possibly discontinuous coefficient. The discontinuous coefficient leads to the fact that neither the standard well-posedness theory of Kruzkhov ([16]) nor the usual numerical methods (see [14]) apply in this case.

Equations of the above type arise while dealing with fluid flows in heterogeneous media such as in two phase flow in a porous medium with changing rock types that arise in the petroleum industry (see [15] for more details), in modeling gravity settling in an ideal clarifier thickener unit used in waste water treatment plants (see 6]), in the modeling of traffic on highways with changing surface conditions (see [23]) and in ion etching in the semiconductor industry (see [24]). A detailed account of various applications is found in [26].

Received by the editor September 19, 2005 and, in revised form, June 23, 2006

2000 Mathematics Subject Classification. Primary 35L65, 65M06, 65M12.

(C)2007 American Mathematical Society Reverts to public domain 28 years from publication 
A special case of equation (11) which is of independent interest is the additive case given by

$$
u_{t}+f(u)_{x}=(k(x))_{x} .
$$

Observe that equation (2) is an example of a conservation law with a conservative source term which can also be singular. The simplest case of equation (11) is the so-called 2-flux case (with a single discontinuity at the origin) given by

$$
\begin{aligned}
u_{t}+(H(x) f(u)+(1-H(x)) g(u))_{x} & =0, \\
u(x, 0) & =u_{0}(x),
\end{aligned}
$$

where $f$ and $g$ are Lipschitz continuous functions and $H$ is the Heaviside function. This case serves as a building block for the analysis of (1), as will be shown in this paper.

As is standard for conservation laws, we will look for weak solutions of equation (11). Even when the coefficient $k$ is Lipschitz, we need to impose additional admissibility criteria or entropy conditions in order to pick out the "correct" solutions. We will need such a notion for the analysis of equation (11). The central problem in the analysis is to develop a suitable concept of entropy solutions for equations of the type (11) and a proof of existence and uniqueness for these entropy solutions. An equally important issue is the development of robust and easy to use numerical methods for (11). We address both these problems during the course of this paper.

Equation (1) has been widely studied from both the theoretical and numerical points of view in recent years. Entropy conditions for some specific cases of (11) were devised by Gimse and Risebro in [12, Diehl in [8, 9, Klingenberg and Risebro in [17 and for the full equation by Karlsen, Risebro and Towers in 20. They used a modified Kruzkhov type entropy condition and showed $L^{1}$ stability of entropy solutions. Concurrently, several existence results for the entropy solutions have been obtained by using regularization of coefficients as in 18, by front tracking as in [12, 17, 18, by explicit Hopf-Lax formulas in [1] and by proving convergence of numerical schemes of the Godunov or Enquist-Osher type as in 2, 25, 27, 28, 19, 6, and the Lax-Friedrichs type as in 21]. Similarly the case of two-phase flow in a medium with changing rock types has been considered in [22] and [15]. The applications relating to the clarifier thickener unit have been considered in [8, 6], etc.

In a recent series of papers [3, 4, 5, the authors have embarked on a program to formulate a proper notion of entropy solutions for (11) and show their existence and uniqueness. In [3, we proposed a new concept of entropy solutions, namely, the Optimal entropy solutions (for (3) with convex type fluxes). This is based on a two-step approach: in the first step, we fix an interface connection $A B$ (see section 2 for definitions) and define an interface entropy condition with respect to this connection. The corresponding $A B$ entropy solutions were shown to be $L^{1}$ contractive for every choice of the interface connection. Existence was proved by showing that a Godunov type scheme converges to the entropy solution. The second step is to optimize a suitable cost functional at the interface, measuring the variation of the solution in an appropriate way involving the connections, and then defining the solution corresponding to the optimal connection as the Optimal entropy solution. In the above paper, we showed the existence and uniqueness of optimal entropy solutions for (3i) with convex type fluxes and found that it agrees 
with entropy solutions developed in [1] and 22. In [4, we considered a changingsign case and developed another notion of generalized entropy solutions (based on generalized Rankine-Hugoniot conditions at the interface). In [5], we treated the case of fluxes having more than one extrema and completed the study of (3).

We had remarked in the above papers that we will use the analysis developed for (31) as building blocks for treating (11), and we proceed to do so in this paper. The aim of this paper is two-fold: first, to extend the entropy framework of [3, 5] to the more general case of equation (1) and show that the entropy solutions exist and are unique. The second aim is to develop and analyze efficient numerical methods for (11), including the key case of conservation laws with singular source terms given by (2).

The entropy framework of this paper is very general, does not impose extra crossing conditions (see [20]) and agrees with the physically observed solutions for flows in heterogenous porous media. The numerical methods developed in this paper are of the aligned discretization type. For the particular case of conservative source terms (and convex fluxes), an aligned discretization based Godunov type scheme was developed for (2) by Greenberg et al in [10. This scheme was shown to work very well on test cases in [10] and was reported to be better than staggered grid schemes in 28]. A proof of convergence of this scheme will be of independent interest. Godunov type schemes are much simpler to implement as compared to the front tracking method and show better resolution than the staggered schemes as pointed out in 28 .

We have organized this paper as follows. In section 2, we propose the entropy framework for (1) and show that the entropy solutions are stable. We describe Godunov type schemes in section 3 and carry out the convergence analysis in section 4. Optimal entropy solutions are described in section 5. In section 6, we present the results of numerical experiments with different schemes.

\section{ENTROPY FRAMEWORK}

To start with, we specify the hypothesis on the fluxes and the coefficient $k$.

Let $-\infty<s<S<+\infty$ and $I=[s, S]$ be the domain of definition of the fluxes. We have the following hypotheses on the coefficient.

$\left(k_{1}\right): k$ is piecewise smooth (say Lipschitz) on $\mathbb{R}$ and has finitely many discontinuities at the points $\left\{a_{m}\right\}$ with $m=1,2, \ldots, M$. We will use the convention that $a_{0}=$ $-\infty$ and $a_{M+1}=\infty$. Furthermore, $k^{\prime}(x)$ is bounded in $D=\mathbb{R} \backslash\left\{a_{m}\right\}_{m=1,2, \ldots, M}$.

$\left(k_{2}\right): T V_{x}(k)<\infty$.

A special case for $k$ with the above hypotheses is when $k$ is piecewise constant. Next, we have the following hypotheses on the flux $f$.

$\left(f_{1}\right): f$ is smooth in both $k$ and $u$. In fact, we need $f$ to be $C^{1,1}$ in the $k$ variable and Lipschitz in the $u$ variable.

$\left(f_{2}\right): f\left(k_{1}, s\right)=f\left(k_{2}, s\right) \quad$ and $\quad f\left(k_{1}, S\right)=f\left(k_{2}, S\right) \quad \forall k_{1}, k_{2} \in \mathbb{R}$.

$\left(f_{3}\right): \forall k \in \mathbb{R}$, the function $u \rightarrow f(k, u)$ has exactly one minimum (resp. maximum) and no maxima (resp. minima) in $[s, S]$.

The hypothesis $f_{3}$ can be generalized to the following:

$\left(\bar{f}_{3}\right): \forall k \in \mathbb{R}$, the function $u \rightarrow f(k, u)$ has finitely many extrema in $[s, S]$.

We remark that $\left(f_{2}\right)$ is sufficient to obtain $L^{\infty}$ bounds on the solution and we can relax it in several cases; see [28, 10] and [3]. For simplicity, we analyze (11) with $\left(f_{3}\right)$ although all the results carry over to the more general class of fluxes in $\left(\bar{f}_{3}\right)$. 
The shape of fluxes with the above hypotheses is shown in Figure 1. Note that the fluxes are allowed to intersect in the interior in an arbitrary manner and can violate the "crossing condition" of [20].

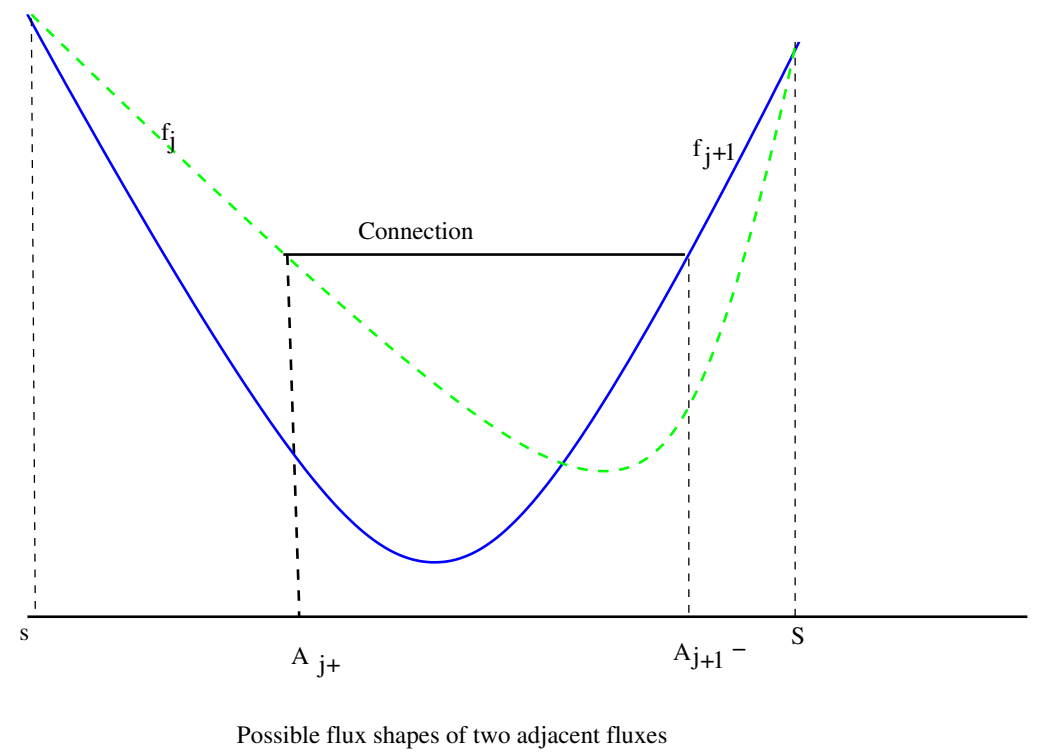

Figure 1. Adjacent flux shapes

Definition 2.1. Weak Solution: $u \in L^{\infty}\left(\mathbb{R} \times \mathbb{R}_{+}\right)$is said to be a weak solution of equation (11) if $\forall \varphi \in C_{c}^{\infty}\left(\mathbb{R} \times \overline{\mathbb{R}}_{+}\right)$, we have that

$$
\int_{\mathbb{R}_{+}} \int_{\mathbb{R}}\left(u \varphi_{t}+f(k(x), u) \varphi_{x}\right) d x d t+\int_{\mathbb{R}} \varphi(0, x) u_{0}(x) d x=0 .
$$

As remarked, we need to impose further admissibility criteria or entropy conditions. Following [3], we start with defining entropy conditions that hold away from the points of discontinuity of $k$. We will use the standard Kruzkhov entropy conditions. Denote the set $D=\mathbb{R} \backslash\left\{a_{m}\right\}, m=1,2, \cdots, M$ that is, the set where the coefficient $k$ is smooth. Then we have

Definition 2.2. Interior Entropy Condition: $u \in L^{\infty}\left(\mathbb{R} \times \mathbb{R}_{+}\right)$is said to satisfy the interior entropy condition provided that $\forall 0 \leq \varphi \in C_{c}^{\infty}\left(D \times \mathbb{R}_{+}\right)$, we have that $\forall c \in \mathbb{R}$,

$$
\begin{gathered}
\int_{\mathbb{R}_{+}} \int_{\mathbb{R}}\left(|u-c| \varphi_{t}+\operatorname{sign}(u-c)(f(k(x), u)-f(k(x), c))\right) \varphi_{x} d x d t \\
\left.-\int_{\mathbb{R}_{+}} \int_{\mathbb{R}} \operatorname{sign}(u-c)(f(k(x), c))_{x} \varphi\right) d x d t \geq 0 .
\end{gathered}
$$

Note that as $k$ is smooth in $D$ and $\varphi$ has compact support contained in $D \times \mathbb{R}_{+}$, $k_{x}$ exists and the above expression makes sense. Interior entropy conditions are not enough to obtain uniqueness, and we need to impose further jump conditions at the 
discontinuities of $k$. As in [3], we use the concept of interface connection defined below. Let $a, b, c, d \in \mathbb{R}, f, g$ be two smooth functions and denote

(6) $\quad I_{a b}(c, d, g, f)=\operatorname{sign}(c-a)(g(c)-g(a))-\operatorname{sign}(d-b)(f(d)-f(b))$.

Denote $f_{m}^{ \pm}=f\left(k\left(a_{m}^{ \pm},.\right)\right.$. By the hypothesis $\left(f_{3}\right)$, for every $m$, the fluxes $f_{m}^{ \pm}$are of the convex type and have exactly one minimum at $\theta_{m}^{ \pm}$. For every $m=1,2, \cdots, M$, the $m$ th interface connection is defined as

Definition 2.3. Interface Connection: For a given $m=1,2, \cdots, M$, let $\left(A_{m}^{-}, A_{m}^{+}\right)$ $\in[s, S]$ be defined as the $m$ th interface connection if the following hold:

1. $f_{m}^{-}\left(A_{m}^{-}\right)=f_{m}^{+}\left(A_{m}^{+}\right)$;

2. $A_{m}^{-} \in\left[s, \theta_{m}^{-}\right]$and $A_{m}^{+} \in\left[\theta_{m}^{+}, S\right]$.

Definition 2.4. Interface Connection Vector: The vector pair $\left(A_{-}, A_{+}\right)$such that $A_{ \pm}=\left(A_{1}^{ \pm}, A_{2}^{ \pm}, \cdots, A_{M}^{ \pm}\right)$is defined to be the interface connection vector if for every $m=1,2, \cdots, M,\left(A_{m}^{-}, A_{m}^{+}\right)$is an interface connection.

We use $A_{ \pm}=\left(A_{-}, A_{+}\right)$to denote a connection henceforth. Some examples of connections are shown in Figure 1. Unlike in [3], the interface connection vector here is a vector pair as each point of discontinuity of the coefficient contributes a component to this vector. Define the interface entropy condition as

Definition 2.5. Interface Entropy Condition: For a given interface connection vector $A_{ \pm}=\left(A_{-}, A_{+}\right)$, a function $u \in L^{\infty}\left(\mathbb{R} \times \mathbb{R}_{+}\right)$satisfies the interface entropy condition provided that the following holds:

1. $\forall x, t, \quad u(x+, t), u(x-, t)$ exists.

2. For each $m=1,2, \cdots, M$, consider the corresponding trace fluxes $f_{m}^{ \pm}$and the connection component $\left(A_{m}^{-}, A_{m}^{+}\right)$. Then the following holds:

$$
I_{A_{m}^{-}, A_{m}^{+}}\left(u\left(a_{m}^{-}, t\right), u\left(a_{m}^{+}, t\right), f_{m}^{-}, f_{m}^{+}\right) \geq 0 \quad \text { a.e. } \quad t,
$$

where $I$ is defined as in (6).

The entropy solutions corresponding to each connection vector are defined as

Definition 2.6. $A_{ \pm}$-Entropy Solution: A function $u \in L^{\infty}\left(\mathbb{R} \times \mathbb{R}_{+}\right)$is defined to be an $A_{ \pm}$-entropy solution of (11) for a given interface connection vector $A_{ \pm}$provided that the following holds:

1. $u$ is a weak solution of (11), i.e., it satisfies (4);

2. $u$ satisfies the interior entropy condition (5);

3. $u$ satisfies the interface entropy condition (7) corresponding to the interface connection vector $A_{ \pm}$.

The above concept of entropy solutions is analogous to the one introduced for the two-flux problem (3) in 3], the only difference being that the interior entropy condition has one extra term in (5) and the interface entropy condition has to take into account the contribution from each point of discontinuity of $k$ and hence is a vector with $M$ components. We have the following stability result.

Theorem 2.1. For any given choice of the interface connection vector $A_{ \pm}$, let $u$ and $v$ be two $A_{ \pm}$entropy solutions corresponding to the initial data $u_{0}$ and $v_{0}$, respectively; then we have the following estimate:

$$
\int_{-\infty}^{+\infty}|u(x, t)-v(x, t)| d x \leq C \int_{-\infty}^{+\infty}\left|u_{0}(x)-v_{0}(x)\right| d x .
$$


Furthermore, if $u_{0} \equiv v_{0}$, then $u \equiv v$ and the $A_{ \pm}$entropy solution is unique. The constant $C$ depends on the derivative of $k$ in the smooth parts of the domain and if $k$ is piecewise constant, then $C=1$ and the solutions are contractive in $L^{1}$.

Proof. The proof will be a consequence of the now standard doubling of variables argument first introduced by Kruzkhov in [16; see 20, and the following crucial comparison lemma.

Lemma 2.1. For any given connection $A_{ \pm}$, let both $u, v$ satisfy the interface entropy condition. Then for every $m=1,2, \cdots, M$ and for almost every $t>0$, we have that

$$
\begin{aligned}
I_{m}(t)= & \operatorname{sign}\left(u\left(a_{m}^{-}, t\right)-v\left(a_{m}^{-}, t\right)\right)\left(f_{m}^{-}\left(u\left(a_{m}^{-}, t\right)\right)-f_{m}^{-}\left(v\left(a_{m}^{+}, t\right)\right)\right) \\
& -\operatorname{sign}\left(u\left(a_{m}^{+}, t\right)-v\left(a_{m}^{+}, t\right)\right)\left(f_{m}^{+}\left(u\left(a_{m}^{+}, t\right)\right)-f_{m}^{+}\left(v\left(a_{m}^{+}, t\right)\right)\right)
\end{aligned}
$$

$$
\geq 0 \text {. }
$$

Proof. For any fixed $m$, the above lemma is just a restatement of Lemma 3.2 of [3], where we have presented a detailed proof. The above inequality is a consequence of the interface entropy condition (7).

Next, we sketch the proof of the theorem below. Since it follows more or less on the lines of the proof of Theorem 2.1 in Karlsen, Risebro and Towers (20]), we will just give the steps and refer the reader to the above reference for details.

Step 1: First take the test functions $0 \leq \varphi \in C_{c}^{\infty}\left(D \times \mathbb{R}_{+}\right)$and use a standard doubling of variables argument as in [16, 20, Appendix A.1] and the interior entropy condition (5) to obtain the following inequality:

$$
\begin{aligned}
\int_{0}^{\infty} & \int_{-\infty}^{\infty}\left(|u-v| \varphi_{t}+\operatorname{sign}(u-v)(f(k, u)-f(k, v)) \varphi_{x}\right) d x d t \\
& \leq C \int_{0}^{\infty} \int_{-\infty}^{\infty}|u-v| \varphi .
\end{aligned}
$$

Step 2: Next we have to relax the assumption that $\varphi$ has support away from the discontinuities of $k$. For that we use the following cut-off function (as in [20]):

$$
\mu_{h}(x)= \begin{cases}\frac{1}{h}(x+2 h), & x \in[-2 h,-h], \\ 1, & x \in[-h, h], \\ \frac{1}{h}(2 h-x), & x \in[h, 2 h], \\ 0, & |x| \geq 2 h .\end{cases}
$$

Then we define $\Psi_{h}=1-\sum_{m=1}^{M} \mu_{h}\left(x-a_{m}\right)$ and note that $\forall \varphi \in C_{c}^{\infty}(\mathbb{R})$. The function $\varphi \Psi_{h}$ is an admissible test function in (10) and, by substituting it in the above inequality and by suitable integration by parts (as in 20]), we get that

$$
\begin{aligned}
\int_{0}^{\infty} & \int_{-\infty}^{\infty}\left(|u-v| \varphi_{t} \Psi_{h} d x d t\right. \\
& \left.+\int_{0}^{\infty} \int_{-\infty}^{\infty} \operatorname{sign}(u-v)(f(k, u)-f(k, v)) \Psi_{h} \varphi_{x}\right) d x d t \\
& +\int_{0}^{\infty} \int_{-\infty}^{\infty} \operatorname{sign}(u-v)(f(k, u)-f(k, v)) \Psi_{h}^{\prime} \varphi d x d t \\
& \leq C \int_{0}^{\infty} \int_{-\infty}^{\infty}|u-v| \varphi \Psi_{h} d x d t .
\end{aligned}
$$


Now by using the fact that $\Psi_{h} \rightarrow 1$ in $L^{1}$ as $h \rightarrow 0$ and by computing the derivatives of the function $\mu_{h}$, we get that (12) reduces to

$$
\begin{aligned}
\int_{0}^{\infty} & \int_{-\infty}^{\infty}|u-v| \varphi_{t} d x d t \\
& +\int_{0}^{\infty} \int_{-\infty}^{\infty} \operatorname{sign}(u-v)(f(k, u)-f(k, v)) \varphi_{x} d x d t \\
& +\int_{0}^{\infty} \sum_{m=1}^{M} I_{m}(t) \varphi(0, t) d t \leq C \int_{0}^{\infty} \int_{-\infty}^{\infty}|u-v| \varphi d x d t .
\end{aligned}
$$

Now by using Lemma 2.1, for each $m$ we get that for all test functions $0 \leq \varphi \in$ $C_{c}^{\infty}\left(\mathbb{R} \times \mathbb{R}_{+}\right)$, the following inequality holds:

$\int_{0}^{\infty} \int_{-\infty}^{\infty}\left(|u-v| \varphi_{t}+\operatorname{sign}(u-v)(f(k, u)-f(k, v)) \varphi_{x}\right) d x d t \leq C \int_{0}^{\infty} \int_{-\infty}^{\infty}|u-v| \varphi$,

thus concluding step 2. Note the key role played by the sign of the $I_{m}(t)$ 's (Lemma 2.1 in the above argument) and the crucial part played by the interface entropy condition in that argument. The fact that we use pointwise interface entropy conditions (7) allows us to control the sign of $I_{m}(t)$ irrespective of whether the fluxes satisfy the "crossing condition" of [20] or not.

Step 3: Once we have the estimate (14) for all test functions, we use the standard argument of Kruzkhov ([16]) of integrating over the cone in order to deduce that

$$
\int_{-\infty}^{+\infty}|u(x, t)-v(x, t)| d x \leq C \int_{-\infty}^{+\infty}\left|u_{0}(x)-v_{0}(x)\right| d x
$$

thus proving the theorem.

It is easy to see that the constant $C$ is equal to 1 when $k$ is piecewise constant, and then we see that for each choice of connection $A_{ \pm}$, the solutions form an $L^{1}$ contractive semigroup; otherwise we only get $L^{1}$ stability. So, for infinitely many classes of connections, we get a stability result in each class and extend the uniqueness results for the two-flux problem (3) to the more general case of (1).

\section{NuMERICAL SCHEMES}

In this section, we describe two numerical schemes of the Godunov finite volume type for (11). In order to do so, we start with some definitions.

Let $h \in \operatorname{Lip}[s, S]$. Then the standard Godunov flux (see [13]) is given by

$$
H(a, b)=\left\{\begin{array}{lll}
\min _{\theta \in[a, b]} h(\theta) & \text { if } \quad a \leq b, \\
\max _{\theta \in[b, a]} h(\theta) & \text { if } \quad a \geq b .
\end{array}\right.
$$

Let $h^{-}, h^{+} \in[s, S]$ be two functions such that they have exactly one minimum and no maxima in $[s, S]$, and let $\theta^{-}, \theta^{+}$be the corresponding minima of the fluxes. Then define

Definition 3.1. Standard Interface Godunov flux: The standard interface Godunov flux is defined as

$$
H^{-,+}(a, b)=\max \left\{H^{-}\left(a, \theta^{-}\right), H^{+}\left(\theta^{+}, b\right)\right\} .
$$


Similarly for a fixed connection $\left(B^{-}, B^{+}\right)$(for definition, see section 2) corresponding to the fluxes $\left(f^{-}, f^{+}\right)$, define

Definition 3.2. $B^{ \pm}$-Interface Godunov Flux: The $B^{ \pm}$-interface Godunov flux is defined by

$$
H_{B}^{-,+}(a, b)=\max \left\{H^{-}\left(a, B^{-}\right), H^{+}\left(B^{+}, b\right)\right\},
$$

where $H^{-}, H^{+}$are the standard Godunov fluxes corresponding to the functions $h^{-}$and $h^{+}$, respectively. Observe that $H^{-,+}=H_{\theta}^{-,+}$. In [3], the above fluxes were shown to be Lipschitz continuous in both variables, nondecreasing in the first variable and nonincreasing in the second variable. The interface fluxes are not consistent, i.e., $H^{-,+}(a, a)$ need not be equal to either $h^{-}(a)$ or $h^{+}(a)$.

We start with the description of the different numerical schemes,

Scheme I. Fix the set of discontinuities of $k=\left\{a_{m}\right\}$ with $m=1,2, \cdots, M$, and discretize $\left[a_{m}, a_{m+1}\right]$ (with $a_{0}=-\infty$ and $a_{M+1}=+\infty$ by convention) uniformly with mesh size $h_{m}$. Let $\bar{h}=\max _{m} h_{m}$ and $\underline{h}=\min _{m} h_{m}$. For simplicity in the notation, we assume that $\bar{h}=\underline{h}=h$ and work with the same mesh size $h$. (This implicitly assumes that the distance between the discontinuities $a_{m}$ is constant. It will be clear from the proofs that the nonuniform mesh size does not create any extra problems in the convergence analysis.) Denote the mesh points as $x_{j-1 / 2}, \quad j \in \mathbb{Z}$ with the convention that $x_{j_{m}-1 / 2}=a_{m}$. Also denote $x_{j}=0.5\left(x_{j-1 / 2}+x_{j+1 / 2}\right)$ as the midpoints of each cell. We discretize time uniformly with time step $\Delta t$ and denote the time mesh points as $t_{n}=n \Delta t$. We restrict the size of the time step by a CFL condition. Let $\lambda=\frac{\Delta t}{h}$,

$$
2 \lambda \bar{M} \leq 1,
$$

where $\bar{M}=\max _{u \in I, k \in \mathbb{R}} \operatorname{Lip}\left(f_{u}(k, u)\right)$. The initial data and the coefficient are discretized as

$$
u_{j}^{0}=\frac{1}{h} \int_{x_{j-1 / 2}}^{x_{j+1 / 2}} u_{0}(x) d x, \quad k_{j}=\left\{\begin{array}{lll}
k\left(x_{j}\right) & \text { if } & x_{j-1 / 2} \leq x<x_{j+1 / 2}, \\
k\left(a_{m}^{+}\right) & \text {if } & x_{j_{m}-1 / 2} \leq x<x_{j_{m}+1 / 2}, \\
k\left(a_{m}^{-}\right) & \text {if } & x_{j_{m}-3 / 2} \leq x<x_{j_{m}-1 / 2} .
\end{array}\right.
$$

The discretization of the data and the coeffcient above are aligned with each other. Define the corresponding fluxes at each cell-center as $f_{j}(u)=f\left(k_{j}, u\right)$. For a fixed $h>0$ the equation (11) reduces to the following:

$$
u_{t}+\left(f_{j}(u)\right)_{x}=0 \quad \text { if } \quad x_{j-1 / 2}<x<x_{j+1 / 2} .
$$

We define Godunov type fluxes at each cell-edge by solving Riemann problems corresponding to "locally" 2-flux problems and average along cells to obtain the finite volume scheme,

$$
u_{j}^{n+1}=\left\{\begin{array}{lll}
u_{j}^{n}-\lambda\left(F^{j, j+1}\left(u_{j}^{n}, u_{j+1}^{n}\right)-F^{j-1, j}\left(u_{j-1}^{n}, u_{j}^{n}\right)\right) & \text { if } j_{m}<j \leq j_{m+1}-1, \\
u_{j}^{n}-\lambda\left(F^{j, j+1}\left(u_{j}^{n}, u_{j+1}^{n}\right)-F_{A_{m}^{ \pm}}^{j-1, j}\left(u_{j-1}^{n}, u_{j}^{n}\right)\right) & \text { if } j=j_{m}, \\
u_{j}^{n}-\lambda\left(F_{A_{m+1}^{j}}^{j, j+1}\left(u_{j}^{n}, u_{j+1}^{n}\right)-F^{j-1, j}\left(u_{j-1}^{n}, u_{j}^{n}\right)\right) & \text { if } j=j_{m+1}-1,
\end{array}\right.
$$

where $F^{j, j+1}$ is the standard Godunov interface flux (16) corresponding to the fluxes $f_{j}$ and $f_{j+1}$ and $F_{A_{m}^{ \pm}}^{j-1, j}$ is the $A_{m}^{ \pm}$-interface Godunov flux (17) corresponding to the fluxes $f_{m}^{ \pm}$. Thus we use the standard Godunov flux away from the discontinuities of 
$k$ and, at the discontinuities $a_{m}$, we replace it with the $A_{m}^{ \pm}$-interface flux in order to obtain consistency with the interface entropy condition. The main differences between (19) and the aligned scheme proposed in [10] are the treatment of a general (not necessarily additive) coefficient, explicit formulas for the interface fluxes and special treatment of the discontinuities of $k$ to obtain consistency with the interface entropy condition. Corresponding piecewise constant functions for the scheme are defined as

$$
u_{h}(x, t)=u_{j}^{n} \quad \text { if } \quad(x, t) \in\left[x_{j-1 / 2}, x_{j+1 / 2}\right) \times[n \Delta t,(n+1) \Delta t) .
$$

It is shown that the total variation of Scheme I (in terms of the singular mapping) is bounded and it satisfies the interior as well as interface entropy conditions. Unfortunately, we are unable to show that it converges as the interface fluxes are not consistent. A simple modification of this scheme leads to a convergent scheme described below.

Scheme II. In scheme I, the discretization of the coefficient $k$ is aligned to that of the unknown. Another class of finite volume methods for (11) are the schemes of Godunov or of Enquist-Osher type developed in [27, 28, 19] that are based on staggering the discretizations of the coefficient and the unknown. This step results in simple schemes that have been shown to converge in several situations. But, as discussed in [28, 25, 15], there are several situations where the aligned schemes perform better. Another disadvantage with the staggered schemes is that these schemes are not "entropy flexible", i.e., we cannot use them to generate $A_{ \pm}$entropy solutions for any choice of the connection $A_{ \pm}$(see [3] for examples). Our objective is to design a scheme that is easy to use, converges and is entropy consistent. Away from the discontinuities, we demand that the solution satisfy the interior entropy condition (5). Next, we describe an alternative scheme (Scheme II), where we combine an aligned discretization of the coefficient and the unknown at the points of discontinuity of $k$ and stagger the discretization of the coefficient and the unknown away from the discontinuities. This mixed method allows us to use the advantages of both the approaches. As we stagger away from the discontinuities, the proof of consistency with the interior entropy condition is straightforward and, using a direct Riemann solver at the discontinuities, we can show consistency with the interface entropy condition and can generate any $A_{ \pm}$-entropy solution for (11). The use of a direct Riemann solver at the discontinuities also allows better numerical performance in some situations (discussed in section 6). Space and time are discretized exactly as in Scheme I, and we denote the mesh points as $x_{j-1 / 2}$. We use the same $C F L$ condition and initialize using the averaging of initial data as in Scheme I. Let $k_{j}, f_{j}$ be as defined for Scheme $\mathbf{I}$. Then we have the following scheme given by

$$
w_{j}^{n+1}= \begin{cases}w_{j}^{n}-\lambda\left(F^{j}\left(w_{j}^{n}, w_{j+1}^{n}\right)-F^{j-1}\left(w_{j-1}^{n}, w_{j}^{n}\right)\right) & \text { if } j_{m}<j \leq j_{m+1}-1, \\ w_{j}^{n}-\lambda\left(F^{j}\left(w_{j}^{n}, w_{j+1}^{n}\right)-F_{A_{m}^{ \pm}}^{j-1, j}\left(w_{j-1}^{n}, w_{j}^{n}\right)\right) & \text { if } j=j_{m} \\ w_{j}^{n}-\lambda\left(F_{A_{m+1}^{j}, j+1}^{ \pm}\left(w_{j}^{n}, w_{j+1}^{n}\right)-F^{j-1}\left(w_{j-1}^{n}, w_{j}^{n}\right)\right) & \text { if } j=j_{m+1}-1\end{cases}
$$

where $F^{j}$ is the standard Godunov flux corresponding to the flux function $f_{j}$ and $F_{A_{m}^{ \pm}}^{j, j+1}$ is the $A_{m}^{ \pm}$-interface Godunov flux (17) corresponding to the flux functions $f_{j}$ 
and $f_{j+1}$. Scheme $\mathbf{I I}$ is exactly like Scheme $\mathbf{I}$ except that away from the discontinuities $a_{m}$, the interface numerical fluxes are replaced by standard fluxes corresponding to the flux functions $f_{j}$. The fluxes $F^{j}$ being standard fluxes are consistent. So we have inconsistent numerical fluxes only at the discontinuities. This crucial modification allows us to prove convergence and consistency with the interior entropy condition. We retain the $A_{m}^{ \pm}$-interface fluxes at the discontinuities. This allows us to show interface entropy consistency and gives better numerical performance. Observe that if $k$ is piecewise constant, then Scheme II coincides with Scheme I.

Another way of obtaining Scheme II is to stagger the coefficient $k$ in $\left(a_{m}, a_{m+1}\right)$ and to align it with the unknown at $a_{m}$. This allows us to combine staggered discretization away from the discontinuities and aligned discretization at the discontinuities, thus enabling us to use the advantage of both approaches. We define the scheme functions as

$$
w_{h}(x, t)=w_{j}^{n} \quad \text { if } \quad(x, t) \in\left[x_{j-1 / 2}, x_{j+1 / 2}\right) \times[n \Delta t,(n+1) \Delta t) .
$$

We show that Scheme II converges and is entropy consistent in section 4 and report numerical experiments that compare the two schemes in section 6 . Define the following estimators of the total variation of the scheme:

$$
\begin{aligned}
N_{1}\left(f, k, u_{0}\right)= & \sup _{h>0} \sum_{m=0}^{M+1}\left(\sum_{j=j_{m}+1}^{j_{m+1}-2}\left|F^{j, j+1}\left(u_{j}^{0}, u_{j+1}^{0}\right)-F^{j-1, j}\left(u_{j-1}^{0}, u_{j}^{0}\right)\right|\right. \\
& +\left|F^{j_{m}, j_{m}+1}\left(u_{j_{m}}^{0}, u_{j_{m}+1}^{0}\right)-F_{A_{m}^{ \pm}}^{j_{m}-1, j_{m}}\left(u_{j_{m}-1}^{0}, u_{j_{m}}^{0}\right)\right| \\
& +\mid F_{A_{m+1}^{ \pm}}^{j_{m+1}-1, j_{m+1}}\left(u_{j_{m+1}-1}^{0}, u_{j_{m+1}}^{0}\right) \\
& \left.-F^{j_{m+1}-2, j_{m+1}-1}\left(u_{j_{m+1}-2}^{0}, u_{j_{m+1}-1}^{0}\right) \mid\right), \\
N_{3}\left(f, k, w_{0}\right)= & \sup _{h>0} \sum_{m=0}^{M+1}\left(\sum_{j=j_{m}+1}^{j_{m+1}-2}\left|F^{j}\left(w_{j}^{0}, w_{j+1}^{0}\right)-F^{j-1}\left(w_{j-1}^{0}, w_{j}^{0}\right)\right|\right. \\
& +\left|F^{j_{m}}\left(w_{j_{m}}^{0}, w_{j_{m}+1}^{0}\right)-F_{A_{m}^{ \pm}}^{j_{m}-1, j_{m}}\left(w_{j_{m}-1}^{0}, w_{j_{m}}^{0}\right)\right| \\
& +\mid F_{A_{m+1}^{ \pm}}^{j_{m+1}-1, j_{m+1}}\left(w_{j_{m+1}-1}^{0}, w_{j_{m+1}}^{0}\right) \\
& \left.-F^{j_{m+1}-2}\left(w_{j_{m+1}-2}^{0}, w_{j_{m+1}-1}^{0}\right) \mid\right) .
\end{aligned}
$$

\section{Convergence analysis}

In this section, we carry out the convergence analysis of Schemes I and II that were described in section 3. We show that Scheme II converges to the entropy solution of (1). This proves that for each choice of connection $A_{ \pm}$, the $A_{ \pm}$-entropy solutions exist and are unique. We also establish $L^{\infty}$ and $T V$ (measured in terms of a singular mapping) bounds.

We start with the following lemma for both schemes.

Lemma 4.1. Let $u_{j}^{n}$ and $w_{j}^{n}$ be defined by Schemes $\mathbf{I}$ and $\mathbf{I I}$, respectively, and let the CFL condition be satisfied. Then the following hold.

(i) The schemes $\mathbf{I}$ and $\mathbf{I I}$ are monotone.

(ii) The schemes are discrete $L^{1}$ contractive i.e., if $u_{0}, \bar{u}_{0} \in L^{\infty}(\mathbb{R},[s, S])$ and if $\left\{u_{j}^{n}\right\},\left\{\bar{u}_{j}^{n}\right\}$ and $\left\{w_{j}^{n}\right\},\left\{\bar{w}_{j}^{n}\right\}$ are the corresponding solutions calculated by 
Schemes $\mathbf{I}$ and $\mathbf{I I}, 2 \lambda \bar{M} \leq 1$ and $i_{0} \leq j_{0}$, then

$$
\begin{aligned}
\sum_{j}\left|u_{j}^{n+1}-u_{j}^{n}\right| & \leq \sum_{j}\left|u_{j}^{n}-u_{j}^{n-1}\right|, \\
\sum_{j}\left|w_{j}^{n+1}-w_{j}^{n}\right| & \leq \sum_{j}\left|w_{j}^{n}-w_{j}^{n-1}\right| .
\end{aligned}
$$

(iii) We have the following $L^{\infty}$ bounds:

$$
s \leq \min \left\{u_{j}^{n}, w_{j}^{n}\right\} \leq \max \left\{u_{j}^{n}, w_{j}^{n}\right\} \leq S \quad \forall i, j \text { and } \forall n .
$$

Proof. (i) follows directly from the fact that the schemes are in conservation form, the CFL condition and the fluxes across each cell being nondecreasing in the first variable and nonincreasing in the second variable. The proof is exactly as in the proof of Lemma 4.2 of [2].

(ii) also follows as in the proof of Lemma 4.4 of 2 from the monotonicity of the scheme and the Crandall-Tartar lemma.

(iii) is a consequence of the monotonicity of the scheme and the consistency of the interface fluxes at the endpoints $s, S$ which give $[s, S]$ as an invariant region for the schemes. The proof is exactly as the proof of Lemma 4.3 of [2]. In order to get the $L^{\infty}$ bounds, we have used the condition that the fluxes intersect at the endpoints. This is a sufficient condition and can be relaxed considerably as in [10, 28] and [4].

The $L^{\infty}$ bounds give that $u_{h} \rightarrow u, w_{h} \rightarrow w$ along subsequences as $h \rightarrow 0$ in the weak-star topology of $L^{\infty}$, but this weak compactness is not enough to pass to the limit in the nonlinear term. For conservation laws with discontinuous coefficients, this is usually done by using a suitable singular mapping to transform the scheme and obtain bounds on the transformed scheme. Since we use a monotone singular mapping, we can invert it to obtain pointwise compactness, which allows us to pass to the limit in the nonlinear term. This program has been carried out for Enquist-Osher type schemes in [27, 28, 19] and for Godunov type schemes for (3) with convex fluxes in 2, with sign changing coefficients in [25, 4] and for general flux geometries in 5]. First, a very general "cell normalized variation inequality" is proved below.

Let $k_{j}, f_{j}$ be as defined in section 3 and $\left\{U_{j}\right\}, j \in \mathbb{Z}$ be a sequence of numbers in $[s, S]$. Let $\left(A_{j}^{+}, A_{j+1}^{-}\right)$be a connection corresponding to the fluxes $f_{j}, f_{j+1}$ respectively. Then define

$$
z_{j}=\int_{A_{j}^{+}}^{U_{j}}\left|f_{j}^{\prime}(\theta)\right| d \theta, \quad z_{j+1}=\int_{A_{j+1}^{-}}^{U_{j+1}}\left|f_{j+1}^{\prime}(\theta)\right| d \theta .
$$

Lemma 4.2. Let $z_{j}, z_{j+1}$ be as defined above. Then the following holds:

$$
\begin{aligned}
-\left(z_{j}-z_{j+1}\right)_{-} & \leq\left|F^{j, j+1}\left(U_{j}, U_{j+1}\right)-F^{j+1, j+2}\left(U_{j+1}, U_{j+2}\right)\right| \\
& +\left|F^{j, j+1}\left(U_{j}, U_{j+1}\right)-F^{j-1, j}\left(U_{j-1}, U_{j}\right)\right|
\end{aligned}
$$

where $F^{i, i+1}, \quad i=j-1, j, j+1$ is the interface Godunov flux corresponding to the fluxes $f_{i}$ and $f_{i+1}$ and $\left(A_{i}^{+}, A_{i+1}^{-}\right), \quad i=j-1, j, j+1$ is a connection corresponding to the fluxes $f_{i}$ and $f_{i+1}$.

Proof. Observe that (25) implies that the variation of the sequence $U_{j}$ under the transformation given above across each cell is bounded by the variation of the fluxes 
of the adjacent cells. For the proof, we have to consider three different cases. For simplicity assume that the pairs of fluxes $f_{j}, f_{j+1}$ belong to the undercompressive case (i.e., the point of intersection in the interior is undercompressive; i.e., let $\alpha \in(s, S)$ be a point of intersection of $f_{j}$ and $f_{j+1}$ and $f_{j}^{\prime}(\alpha)<0, f_{j+1}^{\prime}(\alpha)>0$; see Figure 1 for an illustration and for the notation). This is the hardest case and for the other cases follow similarly.

Case 1: $z_{j} \leq 0, z_{j+1} \geq 0$.

In this case, we get that $U_{j} \leq A_{j}^{+}$and $U_{j+1} \geq A_{j+1}^{-}$; hence it is easy to check that

$$
-\left(z_{j}-z_{j+1}\right)_{-}=f_{j}\left(U_{j}\right)-f_{j}\left(A_{j}^{+}\right)+f_{j+1}\left(U_{j+1}\right)-f_{j+1}\left(A_{j+1}^{-}\right) .
$$

By the definition of the interface Godunov flux (17), we get that

$$
F^{j, j+1}\left(U_{j}, U_{j+1}\right)=f_{j}\left(A_{j}^{+}\right)=f_{j+1}\left(A_{j+1}^{-}\right) .
$$

Now consider the fluxes $f_{j+1}, f_{j+2}$ and let $\left(A_{j+1}^{+}, A_{j+2}^{-}\right)$be a connection for this pair of fluxes. By definition, the interface Godunov flux is given by

$$
F^{j+1, j+2}\left(U_{j+1}, U_{j+2}\right)=\max \left(F^{j+1}\left(U_{j+1}, A_{j+1}^{+}\right), F^{j+2}\left(A_{j+2}^{-}, U_{j+2}\right)\right) .
$$

Therefore, it follows that

$$
\text { for all } U_{j+2} \in[s, S], \quad F^{j+1, j+2}\left(U_{j+1}, U_{j+2}\right) \geq F^{j+1}\left(U_{j+1}, A_{j+1}^{+}\right) .
$$

By the assumptions in this case, $U_{j+1} \in\left[\theta_{j+1}, S\right]$. By definition of a connection, $A_{j+1}^{+} \in\left[s, \theta_{j+1}\right]$. Therefore we have by the monotonicity of the numerical flux and its consistency that

$$
F^{j+1}\left(U_{j+1}, A_{j+1}^{+}\right) \geq F^{j+1}\left(U_{j+1}, U_{j+1}\right)=f_{j+1}\left(U_{j+1}\right) .
$$

Hence, $\forall U_{j+2} \in[s, S]$, we have that

$$
F^{j+1, j+2}\left(U_{j+1}, U_{j+2}\right) \geq f_{j+1}\left(U_{j+1}\right) .
$$

Similarly, consider the fluxes $f_{j-1}, f_{j}$ and let $\left(A_{j-1}^{+}, A_{j}^{-}\right)$be a connection for this pair of fluxes. By definition, the interface Godunov flux is given by

$$
F^{j-1, j}\left(U_{j-1}, U_{j}\right)=\max \left(F^{j-1}\left(U_{j-1}, A_{j-1}^{+}\right), F^{j}\left(A_{j}^{-}, U_{j}\right)\right) .
$$

Therefore, it follows that

$$
\forall U_{j-1} \in[s, S], \quad F^{j-1, j}\left(U_{j-1}, U_{j}\right) \geq F^{j}\left(A_{j}^{-}, U_{j}\right) .
$$

By the assumptions in this case, $U_{j} \in\left[s, \theta_{j}\right]$. By definition of a connection, $A_{j}^{-} \in\left[\theta_{j}, S\right]$. Therefore we have by the monotonicity of the numerical flux and its consistency that

$$
F^{j}\left(A_{j}^{-}, U_{j}\right) \geq F^{j}\left(U_{j}, U_{j}\right)=f_{j}\left(U_{j}\right) .
$$

Hence, $\forall U_{j-1} \in[s, S]$, we have that

$$
F^{j-1, j}\left(U_{j-1}, U_{j}\right) \geq f_{j}\left(U_{j}\right)
$$

Combining the above arguments, we get that, in this case,

$$
\begin{aligned}
-\left(z_{j}-z_{j+1}\right)_{-} & \leq\left|F^{j, j+1}\left(U_{j}, U_{j+1}\right)-F^{j+1, j+2}\left(U_{j+1}, U_{j+2}\right)\right| \\
& +\left|F^{j, j+1}\left(U_{j}, U_{j+1}\right)-F^{j-1, j}\left(U_{j-1}, U_{j}\right)\right|,
\end{aligned}
$$

thus proving the estimate (25) in this case. 
Case 2: $z_{j} \leq z_{j+1} \leq 0$.

In this case, we get that $U_{j} \leq A_{j}^{+}$and $U_{j+1} \leq A_{j+1}^{-}$. Define $\bar{A}_{j+1}^{-} \in\left[s, \theta_{j+1}\right]$ such that $f_{j+1}\left(\bar{A}_{j+1}^{-}\right)=f_{j+1}\left(A_{j+1}^{-}\right)$. We have to consider two subcases in this case given by

Case 2.1: $\bar{A}_{j+1}^{-} \leq U_{j+1} \leq A_{j+1}^{-}$.

In this case, it is easy to see that

$$
F^{j, j+1}\left(U_{j}, U_{j+1}\right)=f_{j}\left(A_{j}^{+}\right)=f_{j+1}\left(A_{j+1}^{-}\right) .
$$

Also it is easy to see that, in this case,

$$
-\left(z_{j}-z_{j+1}\right)_{-} \leq f_{j}\left(U_{j}\right)-f_{j}\left(A_{j}^{+}\right) .
$$

Now the proof of the estimate follows from the estimate (27).

Case 2.2: $U_{j+1}<\bar{A}_{j+1}^{-}$.

In this case, we get that

$$
F^{j, j+1}\left(U_{j}, U_{j+1}\right)=f_{j+1}\left(U_{j+1}\right),
$$

and it is easy to see that

$$
-\left(z_{j}-z_{j+1}\right)_{-} \leq f_{j}\left(U_{j}\right)-f_{j+1}\left(U_{j+1}\right) .
$$

Again, we can invoke the argument in Case 1, i.e., $\forall U_{j-1} \in[s, S]$, we have that $F^{j-1, j}\left(U_{j-1}, U_{j}\right) \geq f_{j}\left(U_{j}\right)$, and conclude the proof of the estimate (25) in this case.

Case 3: $z_{j+1} \geq z_{j} \geq 0$.

In this case, we have that $U_{j} \geq A_{j}^{+}$and $U_{j+1} \geq A_{j+1}^{-}$and the estimate (25) follows exactly as in the proof of case 2 by replacing $U_{j+1}$ by $U_{j}$. We omit the proof in this case. Thus we have established (25) in all cases and complete the proof of Lemma 4.2.

Note that the "cell normalized variation estimate" proved above is more general than similar estimates proved in 2, 3. In the above references, similar estimates involved standard Godunov fluxes on the RHS of (25) whereas the above estimate handles very general interface fluxes in all the adjacent cells. Next, we state (without proof) a very simple lemma below.

Lemma 4.3. Let $\left\{Z_{j}, U_{j}\right\}, j \in \mathbb{Z}$ be a sequence such that

1. $\exists$ constants $D_{1}, D_{2}$ such that

$$
D_{1} \leq Z_{j} \leq D_{2} \quad \forall j \in \mathbb{Z}
$$

2. $\forall j$, the sequence satisfies the following:

$$
\begin{aligned}
-\left(Z_{j}-Z_{j+1}\right)_{-} & \leq\left|F^{j, j+1}\left(U_{j}, U_{j+1}\right)-F^{j+1, j+2}\left(U_{j+1}, U_{j+2}\right)\right| \\
& +\left|F^{j, j+1}\left(U_{j}, U_{j+1}\right)-F^{j-1, j}\left(U_{j-1}, U_{j}\right)\right| .
\end{aligned}
$$

Then we have that

$$
T V\left(Z_{j}\right) \leq 4\left(\sum_{j \in \mathbb{Z}}\left|F^{j, j+1}\left(U_{j}, U_{j+1}\right)-F^{j-1, j}\left(U_{j-1}, U_{j}\right)\right|\right)+D_{2}-D_{1} .
$$

Equipped with the above lemmas, we carry out the convergence analysis for the different schemes. 
Convergence analysis for Scheme II. We show that Scheme II converges to a weak solution of (11) and is consistent with the interior entropy condition (5) as well as the interface entropy condition (7). We use the singular mapping technique for compactness. To start with, define the singular mapping associated with the sequence $\left(w_{h}\right)$ (defined from (21), (22)). For $m=1,2, \cdots, M$, and for some $\alpha \in$ $[s, S]$, let

$$
\psi_{m}\left(x, t, \alpha, w_{h}\right)=\left\{\begin{array}{lll}
\int_{\alpha}^{w_{h}(x, t)}\left|f^{\prime}(k(x), \theta)\right| d \theta & \text { if } & a_{m}<x<a_{m+1}, \\
\int_{\alpha}^{w_{h}(x, t)}\left|f^{\prime}\left(k\left(a_{m}^{+}\right), \theta\right)\right| d \theta & \text { if } \quad x \leq a_{m}, \\
\int_{\alpha}^{w_{h}(x, t)}\left|f^{\prime}\left(k\left(a_{m+1}^{-}\right), \theta\right)\right| d \theta & \text { if } \quad x \geq a_{m+1} .
\end{array}\right.
$$

Note that for $x \in\left(a_{m}, a_{m+1}\right)$, the singular mapping $\psi_{m}$ is monotone and hence invertible and outside this interval, it is constant. We obtain the following variation estimates for each $\psi_{m}$.

Lemma 4.4. Let $w_{h}$ be as defined in (22). Then for all $t>0$ and for any $\alpha \in[s, S]$, we have that for all $m=1,2, \cdots, M$, the following estimate holds:

$$
T V\left(\psi_{m}\left(w_{h}(., t)\right)\right) \leq 4 N_{3}\left(f, k, u_{0}\right)+C T V(k) .
$$

Proof. Fix $t>0$. By definition, let $n$ be such that $t^{n}<t \leq t^{n+1}$. As $w_{h}$ is piecewise constant over each cell, i.e., $w_{h}(x, t)=w_{j}^{n}$, we can drop the index $n$ for notational convenience. Let $\left\{\bar{x}_{i}\right\}, j \in \mathbb{Z}$ be an increasing sequence of points that partitions $\mathbb{R}$. If needed, we refine the partition further such that the following holds: $\forall i \in \mathbb{Z}$, there exists a $j$ such that either

(i) $\bar{x}_{i}, \bar{x}_{i+1} \in\left[x_{j-1 / 2}, x_{j+1 / 2}\right)$ or

(ii) $\bar{x}_{i} \in\left[x_{j-1 / 2}, x_{j+1 / 2}\right)$ and $\bar{x}_{i+1} \in\left[x_{j+1 / 2}, x_{j+3 / 2}\right)$.

In the above, we just demand that the partition be refined such that either all points lie in adjacent cells or that points lie in the same cell for our $h$-spatial discretization. As we fix $\alpha$ and $t$, we denote, for a given $i \in \mathbb{Z}$,

$$
I_{i}=\left|\psi_{m}\left(\bar{x}_{i}, w_{h}\left(\bar{x}_{i}\right)\right)-\psi_{m}\left(\bar{x}_{i+1}, w_{h}\left(\bar{x}_{i+1}\right)\right)\right| .
$$

Note that the definition of $\psi_{m}$ implies that if $\bar{x}_{i+1}<a_{m}$ or $\bar{x}_{i} \geq a_{m+1}$, then $I_{i} \equiv 0$. In the sequel, we assume that either $\bar{x}_{i+1} \geq a_{m}$ or $\bar{x}_{i} \leq a_{m+1}$.

So, we have to consider the following two cases.

Case 1: $\bar{x}_{i}, \bar{x}_{i+1} \in\left[x_{j-1 / 2}, x_{j+1 / 2}\right)$ for some $j \in \mathbb{Z}$.

In this case we have that

$$
\begin{aligned}
I_{i} & =\left|\int_{\alpha_{j}}^{w_{j}}\right| f^{\prime}\left(k\left(\bar{x}_{i}\right), \theta\right)\left|d \theta-\int_{\alpha}^{w_{j}}\right| f^{\prime}\left(k\left(\bar{x}_{i+1}\right), \theta\right)|d \theta| \\
& \leq \int_{\alpha}^{w_{j}}\left|f^{\prime}\left(k\left(\bar{x}_{i}\right), \theta\right)-f^{\prime}\left(k\left(\bar{x}_{i+1}\right), \theta\right)\right| d \theta \\
& \leq C(S-s)\left|k\left(\bar{x}_{i}\right)-k\left(\bar{x}_{i+1}\right)\right|,
\end{aligned}
$$

where the constant $C$ depends only on the $C^{1,1}$ norm of $f$ in $k$.

Case 2: $\bar{x}_{i} \in\left[x_{j-1 / 2}, x_{j+1 / 2}\right)$ and $\bar{x}_{i+1} \in\left[x_{j+1 / 2}, x_{j+3 / 2}\right)$ for some $j \in \mathbb{Z}$.

In this case, we have to consider further subcases given by

Case 2.1: $j \neq j_{m}$ and $j+1 \neq j_{m}$.

We have that

$$
I_{i}=\left|\int_{\alpha}^{w_{j}}\right| f^{\prime}\left(k\left(\bar{x}_{i}\right), \theta\right)\left|d \theta-\int_{\alpha}^{w_{j+1}}\right| f^{\prime}\left(k\left(\bar{x}_{i+1}\right), \theta\right)|d \theta| .
$$

Now, by adding and subtracting suitable terms, we get that

$$
I_{i} \leq I_{i}^{1}+I_{i}^{2}+I_{i}^{3},
$$


where

$$
\begin{aligned}
I_{i}^{1} & =\int_{\alpha}^{w_{j}}\left|f^{\prime}\left(k\left(\bar{x}_{i}\right), \theta\right)-f^{\prime}\left(k\left(x_{j}\right), \theta\right)\right| d \theta \\
& \leq C(S-s)\left|k\left(\bar{x}_{i}\right)-k\left(x_{j}\right)\right|, \\
I_{i}^{3} & =\int_{\alpha}^{w_{j+1}}\left|f^{\prime}\left(k\left(\bar{x}_{i+1}\right), \theta\right)-f^{\prime}\left(k\left(x_{j+1}\right), \theta\right)\right| d \theta \\
& \leq C(S-s)\left|k\left(\bar{x}_{i+1}\right)-k\left(x_{j+1}\right)\right|
\end{aligned}
$$

and

$$
\begin{aligned}
I_{i}^{2} & =\int_{\alpha}^{w_{j}}\left|f^{\prime}\left(k\left(x_{j}\right), \theta\right)\right| d \theta-\int_{\alpha}^{w_{j+1}}\left|f^{\prime}\left(k\left(x_{j+1}\right), \theta\right)\right| d \theta \\
& =I_{i}^{21}+I_{i}^{22}, \\
I_{i}^{21} & \leq \int_{\alpha}^{w_{j+1}}\left|f^{\prime}\left(k\left(x_{j}\right), \theta\right)-f^{\prime}\left(k\left(x_{j+1}\right), \theta\right)\right| d \theta \\
& \leq C(S-s)\left|k\left(x_{j}\right)-k\left(x_{j+1}\right)\right|, \\
I_{i}^{22} & =\int_{w_{j+1}}^{w_{j}}\left|f^{\prime}\left(k\left(x_{j}\right), \theta\right)\right| d \theta .
\end{aligned}
$$

Let $\left(A_{j}^{+}, A_{j+1}^{-}\right)$be a connection corresponding to the fluxes $f_{j}, f_{j+1}$. As we are away from the discontinuities of $k$, we can assume for simplicity that $f_{j}\left(\theta_{j}\right) \geq f_{j+1}\left(\theta_{j+1}\right)$ and that $A_{j}^{+}=\theta_{j}$ (the only nonundercompressive or optimal connection; see [3]). We write $I_{i}^{22}$ as below:

$$
\begin{aligned}
I_{i}^{22} & \leq I_{i}^{221}+I_{i}^{222}+I_{i}^{223}, \\
I_{i}^{221} & =\left|\int_{A_{j}^{+}}^{w_{j}}\right| f^{\prime}\left(k\left(x_{j}\right), \theta\right)\left|d \theta-\int_{A_{j+1}^{-}}^{w_{j+1}}\right| f^{\prime}\left(k\left(x_{j+1}\right), \theta\right)|d \theta|, \\
I_{i}^{222} & \leq \int_{A_{j+1}^{-}}^{w_{j+1}}\left|f^{\prime}\left(k\left(x_{j}\right), \theta\right)-f^{\prime}\left(k\left(x_{j+1}\right), \theta\right)\right| d \theta \\
& \leq C(S-s)\left|k\left(x_{j}\right)-k\left(x_{j+1}\right)\right|, \\
I_{i}^{223} & =\int_{A_{j}^{+}}^{A_{j+1}^{-}}\left|f^{\prime}\left(k\left(x_{j}\right), \theta\right)\right| d \theta .
\end{aligned}
$$

Next, we estimate $I_{i}^{223}$ below as follows: as $A_{j}^{+}=\theta_{j}$,

$$
\begin{aligned}
I_{i}^{223} & \leq\left|f_{j}\left(A_{j}^{+}\right)-f_{j}\left(A_{j+1}^{-}\right)\right| \\
& \leq\left|f_{j}\left(A_{j}^{+}\right)-f_{j+1}\left(A_{j+1}^{-}\right)\right|+\left|f_{j+1}\left(A_{j+1}^{-}\right)-f_{j}\left(A_{j+1}^{-}\right)\right|, \\
\left|f_{j}\left(A_{j}^{+}\right)-f_{j+1}\left(A_{j+1}^{-}\right)\right| & =0 \text { by the Rankine-Hugoniot condition, } \\
\left|f_{j+1}\left(A_{j+1}^{-}\right)-f_{j}\left(A_{j+1}^{-}\right)\right| & =\left|f\left(k\left(x_{j}\right), A_{j+1}^{-}\right)-f\left(k\left(x_{j+1}\right), A_{j+1}^{-}\right)\right| \\
& \leq \bar{C}\left|k\left(x_{j}\right)-k\left(x_{j+1}\right)\right| .
\end{aligned}
$$

Therefore we get that

$$
I_{i}^{223} \leq \bar{C}\left|k\left(x_{j}\right)-k\left(x_{j+1}\right)\right|
$$

where $\bar{C}$ is a constant that just depends on the Lipschitz norm of $f$ in $k$. Next we have 
Case 2.2: Either $j=j_{m}$ or $j=j_{m+1}$.

In this case, as $\psi_{m}$ is piecewise constant outside $\left(a_{m}, a_{m+1}\right)$, we get that this case also reduces to case 1 and we can use estimate (31).

Now, we will sum over all $i$ in the estimates (31)-(36) and use Lemmas 4.2 and 4.3 in order to estimate the crucial $T V$ type term $\sum_{i} I_{i}^{221}$ and we get that

$$
\begin{aligned}
T V\left(\psi_{m}\left(w_{h}\right)\right) \leq & \left|F^{j_{m}-1, j_{m}}\left(w_{j_{m}-1}, w_{j_{m}}\right)-F^{j_{m}}\left(w_{j_{m}}, w_{j_{m}+1}\right)\right| \\
& +\sum_{j=j_{m}}^{j_{m+1}-1}\left|F^{j}\left(w_{j}, w_{j+1}\right)-F^{j+1}\left(w_{j+1}, w_{j+2}\right)\right| \\
& +\sum_{j=j_{m}+1}^{j_{m+1}}\left|F^{j}\left(w_{j}, w_{j+1}\right)-F^{j-1}\left(w_{j-1}, w_{j}\right)\right| \\
& +\left|F^{j_{m+1}-2, j_{m+1}-1}\left(w_{j_{m+1}-1}, w_{j_{m}}\right)-F^{j_{m}}\left(w_{j_{m}}, w_{j_{m}+1}\right)\right|+C T V(k) \\
\leq & \frac{4}{\lambda}\left|w_{j}^{n+1}-w_{j}^{n}\right|+C T V(k) \\
\leq & \frac{4}{\lambda}\left|w_{j}^{1}-w_{j}^{0}\right|+C T V(k) \\
(37) \quad & 4 N_{3}\left(f, k, u_{0}\right)+C T V(k),
\end{aligned}
$$

thus proving the desired estimate.

Note that the constant $C$ depends on the Lipschitz constant of $f$ and $f^{\prime}$. Hence for every $m=1,2, \cdots, M$, we show that the variation of the singular mapping is bounded.

Lemma 4.5. The following estimates hold:

$$
\begin{aligned}
s \leq w_{h} & \leq S \\
\int_{\mathbb{R}}\left|u_{h}(x, t)-u_{h}(x, \tau)\right| d x & \leq N_{3}\left(f, k, u_{0}\right)(2 \Delta t+|t-\tau|) .
\end{aligned}
$$

Proof. The proof follows exactly as in the proof of Lemma 4.6 of [2]. We omit the details.

The main convergence theorem for Scheme II is given below.

Theorem 4.1. Let $\lambda$ be such that $2 \lambda \bar{M}<1$ and let the initial data $u_{0} \in L^{\infty}(\mathbb{R})$ be such that $s \leq u_{0} \leq S$ and $N_{3}\left(f, k, u_{0}\right)<+\infty$. Then for a fixed $h$, let $w_{h}$ be the approximate solution defined by Scheme II (21), (22). Then there exists a subsequence still denoted by $h \rightarrow 0$ such that $w_{h}$ converges a.e. and in $L^{\infty}\left(\mathbb{R}_{+}, L_{\mathrm{loc}}^{1}(\mathbb{R})\right)$ to a function $u$ which is a weak solution of (11) (i.e. it satisfies (41)). Furthermore, $u$ satisfies the interior entropy condition (5). If $u$ is such that the discontinuities of $u$ are a discrete set of Lipschitz curves, then $u$ satisfies the interface entropy condition (7) with respect to the given interface connection $A^{ \pm}$.

Proof. The proof of the above convergence theorem proceeds in the following three steps.

Step 1: Convergence to a weak solution.

In the first step, we will show that the sequence of approximations $w_{h}$ converges a.e. to a function $u$ and it satisfies (4) and hence is a weak solution to (11). Denote

$$
Z_{h}^{m}(x, t)=\psi_{m}\left(x, t, \alpha, w_{h}(x, t)\right) \quad \forall x, t \in \mathbb{R} \times \mathbb{R}_{+} .
$$


Then by Lemma 4.4, for all $m=0,1,2, \cdots, M$ such that $T V\left(Z_{h}^{m}\right)(., t)<\infty$ and by the Rellich compactness theorem, there exists a function $Z^{m}$ such that (along subsequences still denoted by $h), Z_{h}^{m} \rightarrow Z^{m}$ for a.e. $x \in \mathbb{R}$ and for all $t>0$. Using that for each $m, \psi_{m}$ is invertible in $\left(a_{m}, a_{m+1}\right)$, define a function $u$ as follows:

$$
u(x, t)=\psi_{m}^{-1}\left(x, t, \alpha, Z^{m}(x, t)\right) \quad \text { if } \quad x \in\left(a_{m}, a_{m+1}\right), t>0 .
$$

Hence it follows from the definition that $w_{h} \rightarrow u$ a.e. $x$ and for all $t$ (along a subsequence) as $h \rightarrow 0$. Thus, $u$ is a candidate for the weak solution of (1).

We show $u$ satisfies (4). This is done by a straightforward application of the LaxWendroff theorem ([14]) using the a.e. compactness of the approximate solutions, the $L^{1}$ continuity in time estimate (40) and the consistency of the numerical fluxes away from the discontinuities. The details of the proof are similar to those of [2, 19, 27], and we omit them here. The key point to note is that in Scheme II, the numerical fluxes are consistent except at the discontinuities of $k$. This allows us to use the Lax-Wendroff type argument in the proof of convergence. Such a facility is not available in Scheme $\mathbf{I}$.

Step 2: Consistency with the interior entropy condition.

Next, we show that the limit function $u$ satisfies the interior entropy condition (5). The proof is very similar to a proof for consistency with the Kruzkhov type entropy condition presented in Karlsen, Risebro and Towers ([20]), and we sketch the arguments below.

Note that in the definition of the interior entropy condition (5), it is required that the test functions are supported away from the discontinuities of $k$. Hence it is enough to take test functions $\varphi$ which are supported in $\left(a_{m}, a_{m+1}\right)$ for each $m$ and then sum them to obtain (5). The proof proceeds in three steps, namely

Step 2.1:

We have the following cell entropy inequality in $\left(a_{m}, a_{m+1}\right)$ for each $m$ given by

$$
\begin{aligned}
\left|w_{j}^{n+1}-c\right| & \leq\left|w_{j}^{n}-c\right|-\lambda\left(G^{j}\left(w_{j}^{n}, w_{j+1}^{n}\right)-G^{j-1}\left(w_{j-1}^{n}, w_{j}^{n}\right)\right) \\
& \left.-\lambda\left(\operatorname{sign}\left(w_{j}^{n+1}-c\right)\left(f\left(k\left(x_{j+1}\right), c\right)-f\left(k\left(x_{j}\right), c\right)\right)\right)\right),
\end{aligned}
$$

for all $j_{m}+1 \leq j \leq j_{m+1}-2$, where $w_{j}^{n}$ are the approximations generated by Scheme II, for all constants $c \in \mathbb{R}$ and where for all $j \in \mathbb{Z}$,

$$
G^{j}(a, b)=F^{j}(\max (a, c), \max (b, c))-F^{j}(\min (a, c), \min (b, c)),
$$

where $F^{j}$ is the interior Godunov flux corresponding to the flux function $f\left(k\left(x_{j}\right),.\right)$. Note that the above is the standard numerical entropy flux used by Crandall and Majda (see [7]).

The proof of this cell entropy inequality follows exactly as in Lemma 4.1 of [20]. The consistency of the interior flux is heavily used in the proof.

Step 2.2. Since we have the estimate (42) for the approximate solutions, we pass to the limit as $h \rightarrow 0$ (use the a.e. convergence) to get the following.

Claim: Let $u$ be the limit of $w_{h}$ and let $\varphi>0$ be a test function such that $\operatorname{supp}(\varphi) \subset\left(a_{m}, a_{m+1}\right)$. Then the following holds:

$$
\begin{array}{r}
\int_{0}^{\infty} \int_{-\infty}^{+\infty}\left(|u-c| \varphi_{t}+\operatorname{sign}(u-c)(f(k(x), u)-f(k(x), c)) \varphi_{x}\right) d x d t \\
+\int_{0}^{+\infty} \int_{a_{m}}^{a_{m+1}}\left(\left|f(k(x), c)_{x}\right| \varphi d x d t \geq 0\right.
\end{array}
$$


The proof of the estimate (43) is in fact a simple case of a similar estimate in Lemma 4.2 in 20] and follows by using the regularity of the fluxes away from the discontinuity.

Step 2.3. Now, use the above estimate and combine it with Lemma 4.3 of [20], which gives that we can introduce a $\operatorname{sign}(u-c)$ term in (43) in order to conclude that

$$
\begin{array}{r}
\int_{0}^{\infty} \int_{-\infty}^{+\infty}\left(|u-c| \varphi_{t}+\operatorname{sign}(u-c)(f(k(x), u)-f(k(x), c)) \varphi_{x}\right) d x d t \\
+\int_{0}^{+\infty} \int_{a_{m}}^{a_{m+1}}\left(\operatorname{sign}(u-c)\left(f(k(x), c)_{x}\right) \varphi d x d t \geq 0\right.
\end{array}
$$

which proves (5).

Step 3: Consistency with the interface entropy condition.

For a given interface connection $A^{ \pm}$, we show that the limit $u$ satisfies the interface entropy condition (77). This can be done under the extra assumption that $u$ is regular, i.e., the set of discontinuities of $u$ in space-time is a discrete set of Lipschitz curves. The proof follows as in [3].

First, fix a point of discontinuity $a_{m}$ (since the interface entropy condition is local at each point of discontinuity of $k$ ) and let $\left(A_{m}^{-}, A_{m}^{+}\right)$be the corresponding connection. For simplicity, we assume that the fluxes $f_{m}^{+}$and $f_{m}^{-}$are of the undercompressive type. Other cases can be proved similarly. We will use a contradiction argument as used in 2]. As $T V\left(Z^{m}\right)$ is bounded, therefore $Z^{m}(x+, t), Z^{m}(x-, t)$ exist for all $x$, invert the singular mapping to get that $u(x+, t)$ and $u(x, t)$ exist for all $x$ satisfying the first part of the interface entropy condition (77). Suppose $u$ doesn't satisfy the second part of the interface entropy condition (7) at $a_{m}$. Then it is easy to check that there exists a $t_{0}>0$ such either we have

Case 1: $u\left(a_{m}^{-}, t_{0}\right)<A_{m}^{-}$and $u\left(a_{m}^{+}, t_{0}\right)>A_{m}^{+}$or

Case 2: $A_{m}^{-}<u\left(a_{m}^{-}, t_{0}\right)<\bar{A}_{m}^{-}$and $\bar{A}_{m}^{+}<u\left(a_{m}^{+}, t_{0}\right)<A_{m}^{+}$, where $\bar{A}_{m}^{-} \in\left[\theta_{m}^{-}, S\right]$ such that $f_{m}\left(\bar{A}_{m}^{-}\right)=f_{m}\left(A_{m}^{-}\right)$and $\bar{A}_{m}^{+} \in\left[s, \theta_{m}^{+}\right]$such that $f_{m}\left(A_{m}^{+}\right)=$ $f_{m}\left(\bar{A}_{m}^{+}\right)$. For simplicity, we assume that Case 1 holds. Then, we will use a contradiction argument that is very similar to the one first proposed in 2] and for which we need the following lemma,

Lemma 4.6. Let $(\alpha, \beta) \in \mathbb{R}$ be such that $s \leq \alpha<A_{m}^{-}$and $A_{m}^{+}<\beta \leq S$. Let $w_{h}(x, t, \alpha, \beta)$ be the approximate solutions calculated by Scheme II (21),(22) with the initial data given by

$$
\begin{aligned}
w(x, 0, \alpha, \beta) & =\alpha \text { if } \quad x<a_{m}, \\
& =\beta \text { if } \quad x>a_{m}
\end{aligned}
$$

and let as $h \rightarrow 0, w_{h}(x, t, \alpha, \beta) \rightarrow w(x, t, \alpha, \beta)$ (along subsequences still denoted by h) for a.e. $x \in \mathbb{R}$. Then we have that there exists a constant time $t_{(\alpha, \beta)}$ such that the following holds:

$$
\begin{gathered}
\lim _{x \rightarrow a_{m}-} w(x, t, \alpha, \beta)=A_{m}^{-} \quad \text { if } \quad 0<t \leq t_{(\alpha, \beta)}, \\
\lim _{x \rightarrow a_{m}+} w(x, t, \alpha, \beta)=A_{m}^{+} \quad \text { if } \quad 0<t \leq t_{(\alpha, \beta)} .
\end{gathered}
$$


Proof. The proof follows as in the proof of Lemma 4.8 of [2] and is based on the definition of the interface flux and the monotonicity of the scheme. The only difference is that we can get a local-in-time result on account of the finite speed of propagation and the presence of the other discontinuities.

We then repeat the contradiction argument used in the proof of Theorem 3.2 in [2] with the above test function and get a contradiction to the fact that the solution violates the interface entropy condition at $a_{m}$ for each $m$ and hence show consistency with the interface entropy condition. We refer the reader to [2] for the details.

Convergence analysis for Scheme I. For Scheme I, apart from the monotonicity, discrete $L^{1}$ contractivity and $L^{\infty}$ bounds, we have the following lemma.

Lemma 4.7. Let $u_{h}$ be the approximate solutions computed by Scheme I (19), (20) and let the CFL condition $2 \lambda M<1$ be satisfied. Let $u_{0} \in L^{\infty}(\mathbb{R})$ be the initial data with $s \leq u_{0} \leq S$ and $N_{1}\left(f, k, u_{0}\right)<\infty$. Then there exists a function $\bar{u} \in$ $L^{\infty}\left(\mathbb{R} \times \mathbb{R}_{+}\right)$such that $u_{h}$ converges a.e. to $\bar{u}$ (along a subsequence still denoted by $h)$.

Proof. First note that we are just claiming that $u_{h}$ converges a.e. to a function $\bar{u}$ and we are not claiming that this function $\bar{u}$ is a weak solution of (1). The proof is a consequence of a variation bound on the singular mapping. Define the singular mapping as

$$
Y_{h}^{m}=\psi_{m}\left(x, t, \alpha, u_{h}\right),
$$

where $\psi_{m}$ is defined in (29). Thus we use the same singular mapping as in the convergence proof for Scheme II. Similarly, we have the following variation bound.

Claim: For each $m$, we have that

$$
T V\left(\psi_{m}\left(u_{h}\right)\right) \leq 4 N_{1}\left(f, k, u_{0}\right)+D_{1} T V(k),
$$

where $D$ is a constant that only depends on the Lipschitz constant of $f$ and $\frac{\partial f}{\partial k}$. The proof of the above claim is exactly as the proof of Lemma 4.4 and uses the general cell normalized variation estimates (Lemma 4.2) and the discrete $L^{1}$ contractivity in order to obtain the above estimate. Note that the key step is the use of the cell normalized variation inequality (Lemma 4.2) that involves control of the variation of the singular mapping in each cell with interface Godunov fluxes across the adjacent cells.

The remaining part of the proof is the same as that of the first part of Theorem 4.1. and we obtain that $u_{h} \rightarrow \bar{u}$ along a subsequence. In order to proceed further and show that $\bar{u}$ is indeed a weak solution of (1), we need to suitably modify the Lax-Wendroff argument of Theorem 4.1. We have been unable to do so in view of the lack of consistency of the numerical fluxes in every cell. Note that we were able to show convergence for Scheme II as the numerical fluxes were consistent everywhere except on a set of measure zero, which allows us to use the Lax-Wendroff type argument. We would like to remark that although we are not able to show convergence for Scheme $\mathbf{I}$, the fact that we obtain $L^{\infty}$ and $T V$ bounds (measured via the singular mapping) justifies the good numerical performance of the scheme. 


\section{Optimal Entropy solutions}

We have shown the existence of a unique $A^{ \pm}$-entropy solution for every choice of connection $A^{ \pm}$. The next question is which one of these infinitely $L^{1}$ stable classes of solutions is to be singled out as an appropriate solution that is relevant to some physical model. One possible answer to this question was proposed in [3] and consisted of using suitable cost functionals at the interface and their minimizers as the optimal connections. This strategy led to the selection of the optimal entropy solutions that are physically meaningful for several interesting applications such as flows in porous media, etc. We use a similar concept here. Let $u \in L^{\infty}\left(\mathbb{R} \times \mathbb{R}_{+}\right)$ such that $u(x+, t), u(x-, t)$ exist for all $x, t$, and for each $m$ let $f_{m}^{ \pm}=f\left(k\left(a_{m}^{ \pm}\right),.\right)$, and let $\left(A_{m}^{-}, A_{m}^{+}\right)$be a connection associated with the fluxes $f_{m}^{-}, f_{m}^{+}$. Then define

$$
z_{m}^{ \pm}(t)=\int_{A_{m}^{ \pm}}^{u\left(a_{m}^{ \pm}, t\right)}\left|f_{m}^{ \pm \prime}(\theta)\right| d \theta
$$

and

$$
\begin{aligned}
E_{A_{m}^{ \pm}}(t)= & -\left(z_{m}^{-}(t)-z_{m}^{+}(t)\right)_{-}-\left|F_{A_{m}^{ \pm}}^{-,+}\left(u\left(a_{m}^{-}, t\right), u\left(a_{m}^{+}, t\right)\right)-f\left(k\left(a_{m}^{-}\right), u\left(a_{m}^{-}, t\right)\right)\right| \\
& -\left|F_{A_{m}^{ \pm}}^{-,+}\left(u\left(a_{m}^{-}, t\right), u\left(a_{m}^{+}, t\right)\right)-f\left(k\left(a_{m}^{+}\right), u\left(a_{m}^{+}, t\right)\right)\right|,
\end{aligned}
$$

where $F_{A_{m}^{ \pm}}^{-,+}$is the $A_{m}^{ \pm}$interface Godunov flux (17) corresponding to the fluxes $f_{m}^{-}$ and $f_{m}^{+}$and the connection $A_{m}$. Note that the above is a measure of the variation of the solution at a discontinuity $a_{m}$ measured in terms of the singular mapping. We use the same form as in 3$]$.

Definition 5.1. Optimal connection: A connection vector $\bar{A}^{ \pm}$is said to be an optimal connection if for each $m$, the connection component $\left(\bar{A}_{m}^{-}, \bar{A}_{m}^{+}\right)$satisfies the following:

$$
\max _{u\left(a_{m}^{ \pm}, t\right)} E_{\bar{A}_{m}^{ \pm}}(t)=\min _{A_{m}^{ \pm}} \max _{u\left(a_{m}^{ \pm}, t\right)} E_{A_{m}^{ \pm}}(t), \quad \forall t>0 .
$$

As in [3], it is required that the optimal connection is such that its every component minimizes the maximum error over all possible connections.

Lemma 5.1. $\forall m=1,2, \cdots, M$, there exists a unique optimal connection given by

$$
\begin{aligned}
\left(\bar{A}_{m}^{-}, \bar{A}_{m}^{+}\right) & =\left(\theta_{m}^{-}, \bar{\theta}_{m}^{-}\right) \quad \text { if } \quad f\left(k\left(a_{m}^{-}\right), \theta_{m}^{-}\right) \geq f\left(k\left(a_{m}^{+}\right), \theta_{m}^{+}\right) \\
& =\left(\bar{\theta}_{m}^{+}, \theta_{m}^{+}\right) \quad \text { if } \quad f\left(k\left(a_{m}^{-}\right), \theta_{m}^{-}\right)<f\left(k\left(a_{m}^{+}\right), \theta_{m}^{+}\right),
\end{aligned}
$$

where $\theta_{m}^{ \pm}$are the points of minimum of $f_{m}^{ \pm}$and $\bar{\theta}_{m}^{-} \in\left[\theta_{m}^{+}, S\right]$ is such that $f\left(k\left(a_{m}^{-}\right)\right.$, $\left.\theta_{m}^{-}\right)=f\left(k\left(a_{m}^{+}\right), \bar{\theta}_{m}^{-}\right)$and $\bar{\theta}_{m}^{+} \in\left[s, \theta_{m}^{+}\right]$is such that $f\left(k\left(a_{m}^{+}\right), \theta_{m}^{+}\right)=f\left(k\left(a_{m}^{-}\right), \bar{\theta}_{m}^{+}\right)$.

Proof. The proof follows exactly as in the proof of Theorem 5.1 of [3] as the optimizing condition is local at each discontinuity. Thus we show the existence and uniqueness of optimal entropy solutions for (11).

Remark 5.1. In all of the above analysis, we have dealt with fluxes satisfying hypothesis $f_{3}$ (i.e., the fluxes have exactly one minimum (resp. maximum) and no maxima (resp. minima)) in the domain of definition. This case is the most interesting because the fluxes that arise in two-phase flows and in modeling traffic belong to this class. But there are interesting examples where fluxes with multiple extrema 
and sign-changing fluxes also arise. It is natural to extend the theory developed so far to these more general classes of fluxes satisfying hypothesis $\bar{f}_{3}$.

This extension is rather straightforward and is based on the observation that the main ingredient used in the above analysis is the analysis of the two-flux problem (3). A detailed analysis for (3) with sign-changing fluxes has been carried out in [25, 3, 4] and for the multiple extrema in [5]. As in this paper, we can similarly define $A^{ \pm}$-entropy solutions by retaining the same interior entropy condition (5) and suitably modifying the interface entropy condition (7) based on the corresponding interface entropy conditions for (3). Similar Godunov type schemes can be defined and their convergence analysis carried out as in this paper. The only change will be in the explicit formulas for the interface Godunov flux (16), (17). The proof of convergence will be a combination of the ideas of this paper and a Total Variation Lemma like that of Lemma 4.2 which will follow from the arguments of the above quoted papers.

\section{Numerical Results}

In this section, we present results of various numerical experiments conducted for the schemes considered in this paper. In both experiments, we consider (1) with the following coefficients:

$$
\begin{aligned}
& f(k, u)=f(u)-k(x), \quad k(x)= \begin{cases}0, & \text { if } x<-1 \\
\cos ^{2}\left(\frac{\pi x}{2}\right), & \text { if }-1<x<1, \\
0 & \text { if } x>1\end{cases} \\
& f(u)=\frac{1}{2} u^{2} .
\end{aligned}
$$

This example was first considered in [10, and we use it here for comparison.

Experiment 1. We consider (1) with the coefficients given in (46) and with the following initial data:

$$
u(x, 0)=0 \quad \forall x \in \mathbb{R} .
$$

As in 10, for this case, there are no shocks emerging from $x=-1$ and $x=1$ and the exact solution converges to the following steady state:

$$
U_{1}(x)=\left\{\begin{array}{lll}
0 & \text { if } & x \leq-1 \\
\sqrt{2} \cos \left(\frac{\pi x}{2}\right) & \text { if } & -1 \leq x<0 \\
-\sqrt{2} \cos \left(\frac{\pi x}{2}\right) & \text { if } & 0 \leq x<1 \\
0 & \text { if } & x>1
\end{array}\right.
$$

The numerical results for this problem with Schemes $\mathbf{I}$ and II at times $t=0.5$ and $t=3$ are shown in Figure 2. The parameters are $\lambda=0.25$ and $h=0.025$. As shown in the figures, the schemes resolve the solution quite well and the approximate solutions converge to the steady state by the time $t=3$. Schemes $\mathbf{I}$ and II give very similar results in this experiment.

Experiment 2. We use the same fluxes and sources as experiment 1 and the following initial data:

$$
u(0, x)=\left\{\begin{array}{lll}
0 & \text { if } & x<-1 \\
1 & \text { if } & -1<x<1 \\
0 & \text { if } & x>1
\end{array}\right.
$$



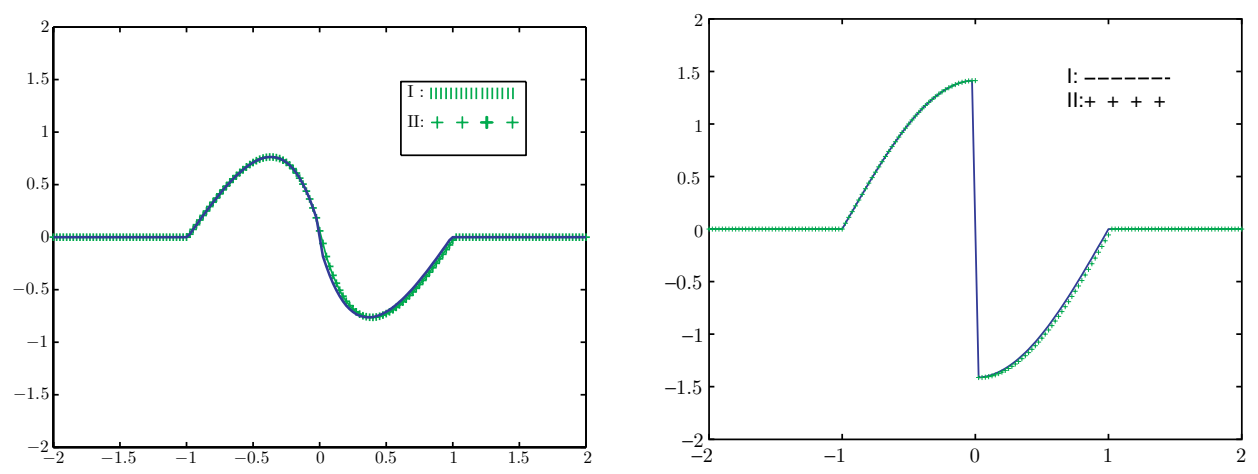

FIGURE 2. Experiment 1 at $t=0.5$ and $t=3$, respectively
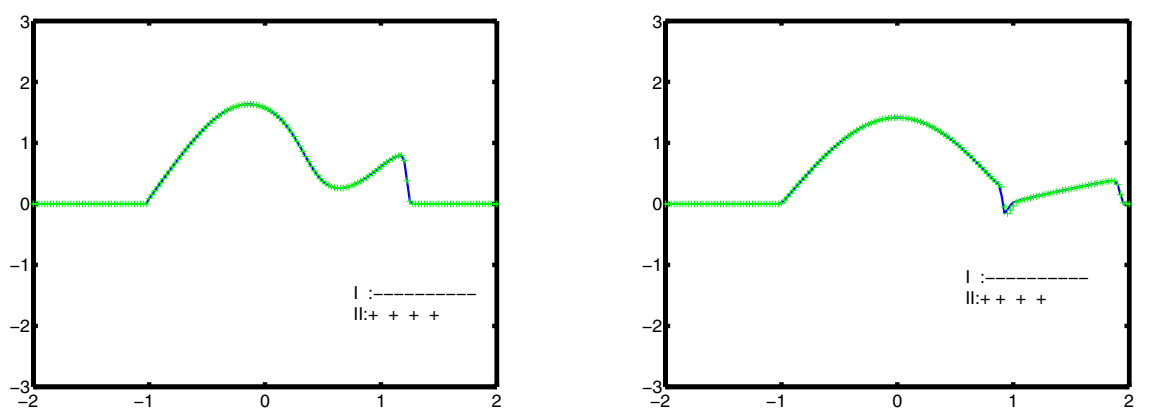

Figure 3. Experiment 2 at $t=0.5$ and $t=3$, respectively

In this case, an exact solution consists of a right moving shock emerging from $x=1$ and the solution converging to the following steady state:

$$
U_{2}(x)=\left\{\begin{array}{lll}
0 & \text { if } & x<-1 \\
\sqrt{2} \cos \left(\frac{\pi x}{2}\right) & \text { if } & -1<x<1, \\
0 & \text { if } & x>1
\end{array}\right.
$$

Again we show results computed with the schemes at times $t=0.5$ and $t=3$ in Figure 3 with the same mesh and CFL parameters as in the first experiment. We remark that we have not used the regridding strategy used in 10 to resolve the shocks.

The computed solutions given by both schemes seem similar and resolve the solutions quite well; particularly the right moving shock is resolved quite well. They converge to the steady state by $t=3$ with the decay of an $N$-wave as reported in 10. We also don't observe any over or undershoots at the right moving shock unlike those reported for a staggered mesh scheme in [28.

From the above numerical experiments and other experiments not reported here, we observe that Schemes I and II are well suited for applications. These schemes are quite easy to implement (on account of explicit formulas for the numerical fluxes) as the staggered mesh schemes of [27, 28] and [19] and show better numerical performance on several test cases by resolving the discontinuities without spurious 
over or undershoots and unphysical traveling waves. At the same time, they are much simpler to implement when compared to the front tracking method.

Comparing Schemes I and II, we can say that although Scheme I performs very well in several situations and we have $L^{\infty}$ and $T V$-type bounds (measured via the singular mapping), we were unable to show convergence for this scheme. On the other hand, Scheme II performs equally well and is slightly easier to implement and has a theoretical basis to its use. We recommend the use of Scheme II for computing solutions of equation (1).

\section{ACKNOWLEDGMENTS}

The authors thank the anonymous referees for their valuable comments and suggestions. Adimurthi and G.D. Veerappa Gowda would like to acknowledge funding from the Indo-French center for promotion of advanced research under the project 3401-2.

\section{REFERENCES}

[1] Adimurthi and G. D. Veerappa Gowda, Conservation Law with Discontinuous flux, Journal of Mathematics, Kyoto Univ., 43(1), 2003, 27-70 MR2028700 (2004k:35248)

[2] Adimurthi, J. Jaffre and G. D. Veerappa Gowda, Godunov type methods for Scalar Conservation Laws with Flux function discontinuous in the space variable, SIAM J. Numer. Anal., 42 (1), 2004, 179-208. MR2051062 (2005c:65066)

[3] Adimurthi, Siddhartha Mishra and G. D. Veerappa Gowda, Optimal entropy solutions for conservation laws with discontinuous flux-functions, J. Hyperbolic Diff. Equations, 2(4), 2005, 783-837. MR2195983

[4] Adimurthi, Siddhartha Mishra and G. D. Veerappa Gowda, Conservation laws with flux function discontinuous in the space variable - II, Discontinuous convex-concave type fluxes and generalised entropy solutions, To appear in J. Comp. Appl. Math.

[5] Adimurthi, Siddhartha Mishra and G. D. Veerappa Gowda, Conservation laws with flux function discontinuous in the space variable - III, The general case, Preprint.

[6] R. Burger, K. H. Karlsen, N. H. Risebro and J. D. Towers, Well-posedness in BV $V_{t}$ and convergence of a difference scheme for continuous sedimentation in ideal clarifier thickener units, Numer. Math, 97(1), 2004, 25-65. MR2045458 (2004m:35175)

[7] M. G. Crandall and A. Majda, Monotone Difference Approximations for scalar conservation laws, Math. Comp. 34 (1980), 385-314. MR551288 (81b:65079)

[8] S. DieHL, On scalar conservation laws with point source and discontinuous flux function modeling continuous sedimentation, SIAM J. Math. Anal., 26(6) (1995), 1425-1451. MR.1356452 (97a:35144)

[9] S. DieHL, A conservation law with point source and discontinuous flux function modeling continuous sedimentation, SIAM J. Appl. Math., 56(2) (1996), 388-419. MR.1381652 (97a:35145)

[10] J. M. Greenberg, A. Y. Leroux, R. Baraille and A. Noussair, Analysis and Approximation of conservation laws with source terms, SIAM J. Numer. Anal., 34(5) (1995), 1980-2007. MR1472206 (98k:65049)

[11] T. Gimse AND N. H. Risebro, Riemann problems with discontinuous flux function, In Proc. 3rd Internat. Conf. Hyperbolic problems Studentlitteratur, Uppsala, 1991, 488-502. MR.1109304 (92b:35094)

[12] T. Gimse AND N. H. Risebro, Solution of Cauchy problem for a conservation law with discontinuous flux function, SIAM J. Math. Anal, 23(3), (1992), 635-648. MR.1158825 (93e:35070)

[13] S. Godunov, Finite difference methods for numerical computation of discontinuous solutions of the equations of fluid dynamics, Math.Sbornik, 47 (1959), 271-306.

[14] E. Godlewski AND P.A. Raviart, Hyperbolic Systems of Conservation Laws, Mathématiques et Applications, Ellipses, Paris, 1991. MR.1304494 (95i:65146)

[15] Jerome Jaffre and Siddhartha Mishra, On the upstream mobility flux scheme for simulating two phase flow in heterogeneous porous media, Preprint. 
[16] S. N. KRuzkhov, First order quasilinear equations in several independent variables, Mat. $\mathrm{Sb}, 10(1970), 217-243$

[17] C. Klingenberg and N. H. Risebro, Convex conservation laws with discontinuous coeffcients: Existence, uniqueness and Asymptotic behavior, Comm. Part. Diff. Eqns, 20 (1995), no 11-12, 1959-1990. MR1361727(96i:35082)

[18] K. H. Karlsen, N. H. Risebro And J. D. Towers, On a nonlinear degenerate parabolic transport-diffusion equation with discontinuous coefficient, Electron. J. Differential Equations, (2002), no 93, 1-23. MR.1938389(2003k:35126)

[19] K. H. Karlsen, N. H. Risebro And J. D. Towers, Upwind difference approximations for degenerate parabolic convection-diffusion equations with a discontinuous coefficient, IMA J. Numer. Anal., 22(4), (2002), 623-664. MR 1937244 (2003i:65071)

[20] K. H. Karlsen, N. H. Risebro and J. D. Towers, $L^{1}$ stability for entropy solution of nonlinear degenerate parabolic convection-diffusion equations with discontinuous coefficients, Skr. K. Nor. Vidensk. Selsk., no 3, (2003) 49 pages. MR2024741 (2004j:35149)

[21] K. H. Karlsen And J. D. Towers, Convergence of the Lax-Friedrichs scheme and stability of conservation laws with a discontinuous time-dependent flux, Chinese Ann. Math. Ser. B., 25(3), (2004), 287-318. MR2086124 (2005h:65145)

[22] E. KAASSChIETER, Solving the Buckley-Leverret equation with gravity in a heterogeneous porous media, Computational Geosciences, 3 (1999), 23-48. MR.1696184 (2000b:76103)

[23] S. Mochon, An analysis for the traffic on highways with changing surface conditions, Math. Model., 9 (1987), 1-11. MR898784 (88e:90032)

[24] D. S. Ross, Two new moving boundary problems for scalar conservation laws, Comm. Pure. Appl. Math 41 (1988) 725-737. MR948078 (89f:35132)

[25] Siddhartha Mishra, Convergence of upwind finite difference schemes for a scalar conservation law with indefinite discontinuities in the flux function, SIAM J. Num. Anal., 43(2), (2005), 559-577. MR2177880 (2006g:65131)

[26] Siddhartha Mishra, Analysis and Numerical approximation of conservation laws with discontinuous coefficients, Ph.D. thesis, Indian Institute of Science, 2005.

[27] J. D. Towers, Convergence of a difference scheme for conservation laws with a discontinuous flux, SIAM J. Numer. Anal., 38(2), (2000), 681-698. MR1770068(2001f:65098)

[28] J. D. Towers, A difference scheme for conservation laws with a discontinuous flux: the nonconvex case, SIAM J. Numer. Anal., 39(4), (2001), 1197-1218. MR1870839|(2002k:65131)

TIFR CENTER, P.O. Box 1234, Bangalore 560012, India

E-mail address: aditi@math.tifrbng.res.in

Center of Mathematics for Applications, University of Oslo, P.O. Box 1053, OSLO-0316, NORWAY

E-mail address: siddharm@cma.uio.no

TIFR CENTER, P.O.Box 1234, BANGAlORE 560012, India

E-mail address: gowda@math.tifrbng.res.in 